\title{
ANTIAPOPTOTIC EFFECT OF PHENYLACETIC ACID AMIDE DERIVATIVES. EVALUATION OF THE STRUCTURE-ACTIVITY RELATIONSHIP
}

\author{
A.A. Ladyka, A.V. Sosnovskaya, M.V. Chernikov, D.I. Pozdnyakov \\ Department of Pharmacology with a course of Clinical Pharmacology, Pyatigorsk Medical \\ and Pharmaceutical Institute-branch of Volgograde state medical university, \\ 357532, Russia, Stavropol Territory, Pyatigorsk, Kalinina ave., 11.
}

DOI: 10.19163/MedChemRussia2021-2021-363

E-mail: a.a.ladyka@yandex.ru

To date, it has been established that the activation of apoptosis underlies a large range of pathological processes, including ischemic events. At the same time, the apoptotic cascade is a promising pharmacotherapeutic target [1]. The antiapoptotic properties of phenylacetic acid amide derivatives (6 compounds, the general formula is shown in Figure 1) were evaluated on a model of focal cerebral ischemia reproduced by the method of Tamura, 1981 [2], by evaluating the changes in the concentration of active isoforms of caspase- 3 and endonuclease $\mathrm{G}$ in the brain supernatant.

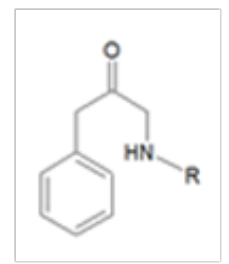

Fig. 1. The general formula of the studied compounds

The relationship between the severity of the pharmacological effect and the structure of the compound was studied by determining the correlation dependencies of the activity and quantum chemical parameters. The dependence of the effect on the minimum docking energy was also evaluated (Molegro 6.0 Virtual Docker software). During the study, it was found that the administration of the studied compounds at a dose of $25 \mathrm{mg} / \mathrm{kg}$ (orally), contributed to a decrease in the concentration of caspase- 3 and endonuclease $g$ in the brain supernatant in relation to animals that did not receive pharmacological support. At the same time, the caspase-inhibitory effect of the studied compounds was most correlated with the ionization potential $(r=0.97384)$, the HUMO energy $(r=0.95588)$, and the minimum docking energy $(r=0.84311)$. The suppression of endonuclease $G$ activity observed in the application of the studied substances directly correlated with the dipole moment $(r=0.9232)$ and the minimum docking energy $(r=0.88947)$.

\section{References}

[1] D. Radak, N. Katsiki, I. Rezanovich, A. Jovanovich, E. Sudar-Milovanovich, S. Zafirovich, S. A. Musad, E. R. Isenovich, Curr Vasc Pharmacol, 2017, 15(2), 115-122

[2] A. Tamura, D. I. Graham, J. McCulloch, G. M. Teasdale, J Cereb Blood Flow Metab, $1981,1(1), 53-60$. 